ESAIM: PROCEEDINGS AND SURVEYS, March 2016, Vol. 53, p. 38-48

M. Campos Pinto and F. Charles, Editors

\title{
ADAPTIVE WAVELET SIMULATION OF WEAKLY COMPRESSIBLE FLOW IN A CHANNEL WITH A SUDDENLY EXPANDED SECTION
}

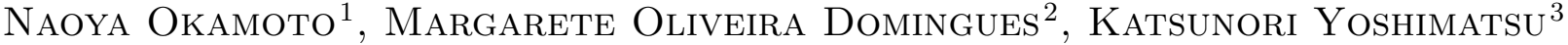 \\ AND KAI SCHNEIDER ${ }^{4}$
}

\begin{abstract}
We present an adaptive multiresolution simulation method for computing weakly compressible flow bounded by solid walls of arbitrary shape, using a finite volume (FV) approach coupled with wavelets for grid adaptation. A volume penalization method is employed to compute the flow in the Cartesian geometry and to impose the boundary conditions. A dynamical adaption strategy to advance both the locally refined grid and the flow in time uses biorthogonal wavelet transforms at each time step. We assess the quality and efficiency of the method for a two-dimensional flow in a periodic channel with a suddenly expanded section. The results are compared with a reference flow obtained by a non-adaptive FV simulation on a uniform grid. It is shown that the adaptive method allows for substantial reduction of CPU time and memory, while preserving the time evolution of the velocity field obtained with the non-adaptive simulation.

Résumé. Nous développons une méthode de simulation multi-résolution adaptative pour calculer les écoulements faiblement compressibles délimités par des parois solides de forme arbitraire, en utilisant des ondelettes et une approcheen volume fini $(\mathrm{FV})$. La pénalisation en volume est employée pour calculer l'écoulement dans une géométrie cartésienne. Une stratégie d'adaptation dynamique pour avancer à la fois la grille raffinée localement et le flux en temps utilise les ondelettes biorthogonales à chaque pas de temps. On évalue la qualité et l'efficacité de la méthode pour un écoulement à deux dimensions dans un canal avec une section soudainement élargie, en comparant avec un calcul de référence obtenu par une méthode en FV non adaptatif. Il est montré que la méthode adaptative permet une réduction substantielle du temps de calcul, tout en préservant l'évolution temporelle du champ de vitesse obtenu avec la simulation non adaptatif.
\end{abstract}

\section{INTRODUCTION}

Wall-bounded flows in complex geometries are often encountered in our daily life and in many engineering applications. The development of computational methods for simulating such flows has been one of the main issues in computational fluid dynamics.

\footnotetext{
${ }^{1}$ Center for Computational Science, Nagoya University, Nagoya, 464-8603, Japan (e-mail: okamoto@ccs.engg.nagoya-u.ac.jp)

${ }^{2}$ Laboratório Associado de Computação e Matemática Aplicada, Coordenadoria dos Laboratórios Associados, Instituto Nacional de Pesquisas Espaciais, Av. dos Astronautas 1758, 12227-010 São José dos Campos, São Paulo, Brazil

(e-mail: margarete.domingues@inpe.br)

${ }^{3}$ EcoTopia Science Institute, Nagoya University, Nagoya, 464-8603, Japan (e-mail: yoshimatsu.katsunori@g.mbox.nagoya-u.ac.jp)

4 M2P2-CNRS \& CMI Aix-Marseille Université, 38 rue F. Joliot-Curie, 13451 Marseille Cedex 20, France

(e-mail: kschneid@cmi.univ-mrs.fr)
}

(C) EDP Sciences, SMAI 2016 
Volume penalization (VP) methods are powerful tools to compute wall-bounded flows in complex geometries or flows with obstacle, while using simple Cartesian geometries. VP methods have been used in the context of incompressible, e.g. [1], or compressible flows, e.g. [2,3]. For a recent review on VP we refer to [4].

Adaptive numerical simulation methods aim to reduce the computational cost, i.e., CPU time and memory, compared to non-adaptive simulation methods, while preserving the accuracy. Adaptive methods track the time evolution of the flow field using a locally refined mesh which changes in time. The number of grid points in the refined mesh is directly related to the computational cost of the methods. The adaptive methods can be more efficient using wavelet representations, because wavelets have attractive mathematical features, e.g. efficient multiscale decomposition and local error indicators [1]. A comparison of an adaptive wavelet method with adaptive mesh refinement both using finite volume discretization has been presented in $[5,6]$ for two- and three-dimensional compressible Euler equations. Wavelet methods in computational fluid dynamics have been reviewed in $[7,8]$.

In order to reduce the number of mesh points not only in the fluid region but also inside the obstacles or walls, adaptive simulation methods coupled with the VP method seem to be promising. In this study we consider weakly compressible flow bounded by solid walls of arbitrary shape, e.g., a compressible flow in micro channels [9]. Our aim is to develop an adaptive wavelet method to compute the flow bounded by complex geometries, employing a VP method for compressible flow [3]. A finite volume (FV) method is used for spatial discretization. The quality and efficiency of the wavelet method is assessed for the flow in a channel with a suddenly expanded section, comparing with a reference flow obtained by a non-adaptive FV simulation method. The paper is organized as follows: after recalling the governing equations, we present the numerical method in section 3. Section 4 shows the numerical results, and conclusions are drawn in section 5 .

\section{BASIC EQUATIONS}

In this section, we recall the penalized Navier-Stokes equations for compressible flow and the corresponding wavelet filtered equations.

\subsection{Penalized Navier-Stokes equations for compressible flows}

The volume penalization method is based on the idea of modeling a solid domain as a porous medium whose permeability tends to zero [10]. The method allows us to consider solid walls of arbitrary shape. A sketch of the flow region $\Omega_{f}$ bounded by solid regions $\Omega_{s}$ is given in Fig. 1. Compressible flow can be modeled by the

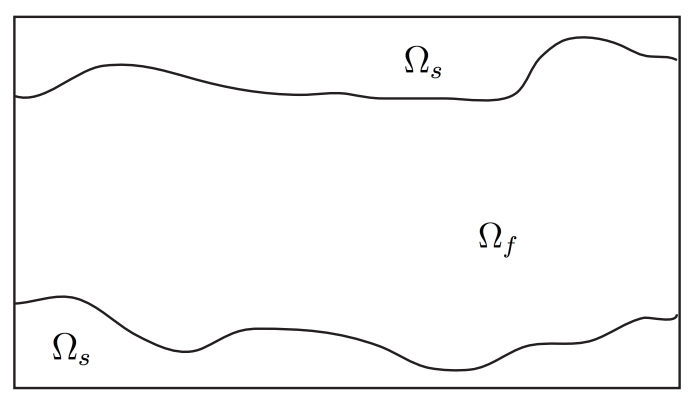

FIgURE 1. Sketch of the computational domain. The fluid region $\Omega_{f}$ is surrounded by solid regions $\Omega_{s}$. The computational domain, a rectangle, is $\Omega=\Omega_{f}+\Omega_{s}$. 
dimensionless penalized Navier-Stokes equations [3],

$$
\begin{aligned}
\frac{\partial \rho}{\partial t} & =-\frac{\partial}{\partial x_{j}}\left(\rho u_{j}\right) \\
\frac{\partial\left(\rho u_{i}\right)}{\partial t} & =-\frac{\partial}{\partial x_{j}}\left(\rho u_{i} u_{j}+p \delta_{i j}-\tau_{i j}\right)-\frac{\chi}{\eta} \rho u_{i}+\rho F_{i}, \\
\frac{\partial(\rho E)}{\partial t} & =-\frac{\partial}{\partial x_{j}}\left[(\rho E+p) u_{j}-u_{i} \tau_{i j}-\lambda \frac{\partial T}{\partial x_{j}}\right]-\frac{\chi}{\eta}\left(\rho E-\rho E_{\Omega}\right)+\rho u_{i} F_{i}, \\
p & =\frac{\rho T}{\gamma M a^{2}}
\end{aligned}
$$

where

$$
E=\frac{p}{(\gamma-1) \rho}+\frac{1}{2} u_{j} u_{j}, \quad \tau_{i j}=\frac{\mu}{R e}\left(\frac{\partial u_{i}}{\partial x_{j}}+\frac{\partial u_{j}}{\partial x_{i}}-\frac{2}{3} \frac{\partial u_{k}}{\partial x_{k}} \delta_{i j}\right), \quad \text { and } \quad \lambda=\frac{\mu}{(\gamma-1) M a^{2} \operatorname{RePr}} .
$$

Here, $u_{i}(\boldsymbol{x}, t)$ is the $i$-th component of velocity, $\rho(\boldsymbol{x}, t)$ the density, $p(\boldsymbol{x}, t)$ the pressure, $T(\boldsymbol{x}, t)$ the temperature, $E(\boldsymbol{x}, t)$ the total energy, $\tau_{i j}(\boldsymbol{x}, t)$ the viscous strain tensor, $\delta_{i j}$ the Kronecker delta, $F_{i}(\boldsymbol{x})$ a forcing term, $\mu(\boldsymbol{x}, t)$ the molecular viscosity, $\lambda$ the conductivity, $\gamma$ the specific heat ratio, $R e$ the Reynolds number, $M a$ the Mach number, $\operatorname{Pr}$ the Prandtl number, $t$ time and $\boldsymbol{x}=\left(x_{1}, x_{2}\right)$. Einstein's summation convention is used for repeated indices. The viscosity $\mu$ obeys the dimensionless Sutherland law $\mu=T^{3 / 2}\left(1+T_{s}\right) /\left(T+T_{s}\right)$, where $T_{s} \approx 0.404$. The permeability of the solid region $\Omega_{s}$ is denoted by $\eta$ and called penalization parameter. The mask function

$$
\chi(\boldsymbol{x})= \begin{cases}1 & \text { in } \Omega_{\mathrm{s}}, \\ 0 & \text { in } \Omega_{\mathrm{f}},\end{cases}
$$

describes the geometry of the flow and the solid domain. The energy of $\Omega_{s}$ is given as $E_{\Omega}=T_{\Omega} /\left[\gamma(\gamma-1) M a^{2}\right]$, where $T_{\Omega}$ is a constant wall temperature. The no-slip condition (i.e., both the parallel and normal components of velocity vanish on the wall) and the isothermal condition on the walls are included in the penalization terms. The Navier-Stokes equations are solved numerically in a periodic computational domain, which consists of the fluid region $\Omega_{f}$ and the solid region $\Omega_{s}$, as illustrated in Fig. 1. In Eqs. (1) - (5), and hereafter, we omit the arguments $\boldsymbol{x}$ and $t$, if not otherwise stated. For convenience, we define a vector $\boldsymbol{U}=\left(\rho, \rho u_{1}, \rho u_{2}, \rho E\right)$.

\subsection{Wavelet filtered equations}

We make a brief summary of wavelets and an adaptive wavelet simulation method for compressible flow, which is called CVS [11]. We then derive a corresponding formulation of the penalized CVS.

Wavelet analysis is an efficient tool to perform multiscale decompositions, since wavelets are well localized functions in space, scale, and direction [12]. Analysis of incompressible, e.g. [13-15], and compressible flow [11] show that coherent structures can be efficiently extracted and the statistics of the original fields is well preserved with a reduced set of degrees of freedom.

The underlying idea of CVS for compressible flow is to decompose $\boldsymbol{U}$ into an organized coherent part $\overline{\boldsymbol{U}}$ and a noise-like incoherent part $\boldsymbol{U}^{\prime}$ using here a biorthogonal wavelet representation at each time step. The coherent part $\overline{\boldsymbol{U}}$ is reconstructed from the few wavelet coefficients whose modulus is larger than a given threshold, while the incoherent part $\boldsymbol{U}^{\prime}$ is obtained from most of the remaining wavelet coefficients. We only compute the time evolution of $\overline{\boldsymbol{U}}$ deterministically, while neglecting the influence of the incoherent part here. The threshold $\epsilon$ used here is based on the $H^{1}$-norm [11]. In the two dimensional case, $\epsilon$ takes a constant value and is not level dependent. It is applied to all components of $\boldsymbol{U}$. For a discussion on the choice of the threshold using other norms, we refer the reader to [11]. 
Following Roussel \& Schneider [11], the dimensionless density $\rho$ and the pressure $p$ are decomposed into

$$
\rho=\bar{\rho}+\rho^{\prime}, \quad p=\bar{p}+p^{\prime}
$$

where ${ }^{-}$and ' respectively denote the coherent and incoherent part. The other remaining variables $\phi$, e.g., the velocity $\left(u_{1}, u_{2}\right)$, the temperature $T$ and energy $E$, are decomposed using the Favre averaging technique, such that

$$
\phi=\bar{\phi}+\phi^{\prime}, \quad \bar{\phi}=\frac{\overline{\rho \phi}}{\bar{\rho}} .
$$

Inserting the decompositions of Eqs. (7) and (8) into Eqs. (1)-(4), while retaining only the coherent part and using the approximation $\bar{\mu}=\mu(\bar{T})$, we obtain the evolution equations for $\overline{\boldsymbol{U}}$, which are called the wavelet filtered equations,

$$
\begin{aligned}
\frac{\partial \bar{\rho}}{\partial t} & =-\frac{\partial}{\partial x_{j}}\left(\bar{\rho} \bar{u}_{j}\right) \\
\frac{\partial\left(\bar{\rho} \bar{u}_{i}\right)}{\partial t} & =-\frac{\partial}{\partial x_{j}}\left(\bar{\rho} \bar{u}_{i} \bar{u}_{j}+\bar{p} \delta_{i j}-\bar{\tau}_{i j}\right)-\frac{\chi}{\eta} \bar{\rho} \bar{u}_{i}+\bar{\rho} F_{i} \\
\frac{\partial(\bar{\rho} \bar{E})}{\partial t} & =-\frac{\partial}{\partial x_{j}}\left[(\bar{\rho} \bar{E}+\bar{p}) \bar{u}_{j}-\bar{u}_{i} \bar{\tau}_{i j}-\bar{\lambda} \frac{\partial \bar{T}}{\partial x_{j}}\right]-\frac{\chi}{\eta}\left(\bar{\rho} \bar{E}-\bar{\rho} E_{\Omega}\right)+\bar{\rho} \bar{u}_{i} F_{i} \\
\bar{p} & =\frac{\bar{\rho} \bar{T}}{\gamma M a^{2}} .
\end{aligned}
$$

\section{NumERICAL SCHEME}

We briefly describe the numerical methods for the penalized Navier-Stokes equations, Eqs. (1) - (4), and the wavelet filtered equations, Eqs. (9) - (12). The discretizations are the same as in Ref. [11] except the treatment of the penalization and forcing terms.

\subsection{Numerical method for the penalized Navier-Stokes equations}

We obtain the reference flow, performing non-adaptive simulations solving Eqs. (1) - (4) with a finite volume (FV) method on a uniform grid, which we call penalized Navier-Stokes simulation (abbreviated as PNS here). We use an explicit $(2,4)$ MacCormack scheme, which is second-order accurate in time, fourth-order in space for the convective terms, and second-order in space for the diffusive terms. The penalization and forcing terms are integrated in time in the same way as the diffusive terms.

The time increment $\Delta t$ is determined, taking into account the Courant-Friedrichs-Lewy condition and its stability constraints which originate from the penalization terms and the diffusion terms. The explicit discretization of the penalization terms requires that $\Delta t$ has to be smaller than $\eta$, see, e.g., the discussion in [16].

\subsection{Numerical method for the wavelet filtered equations}

The time integration of the wavelet filtered equations is performed on an adaptive grid with a tree data structure, a quad-tree here. The adaptive grid is constructed by adding or removing cells, applying thresholding of the wavelet coefficients, which are computed through the cell-averaged multiresolution analysis. A sketch of the adaptive grid with three hierarchy levels is shown in Fig. 2. In the grid in Fig. 2 (b), there are adjacent cells whose scales are different from each other. For the conservative flux computation between these cells, virtual cells indicated by dashed lines are used as illustrated in Fig. 2 (c). The numerical method for the wavelet filtered equations is the same as the one mentioned in Sec. 3.1, except that the computational procedure is now executed on the adaptive grid. A CVS computation with the threshold value $\epsilon=0$ corresponds to the non-adaptive computation, whose grid at the finest level is identical to the grid used in the PNS computation. However, it is not efficient in terms of computational cost and memory due to the overhead. 
(a)

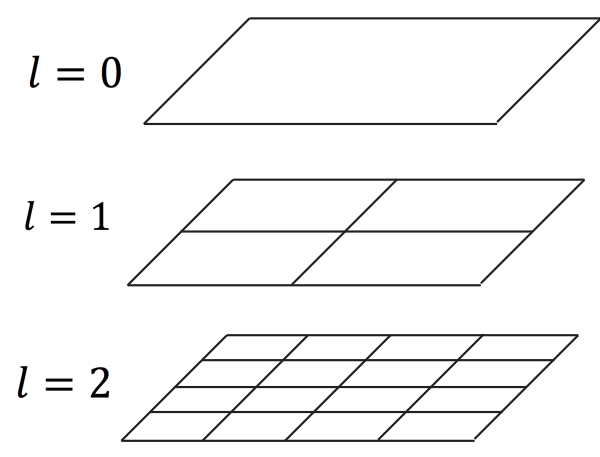

(b)

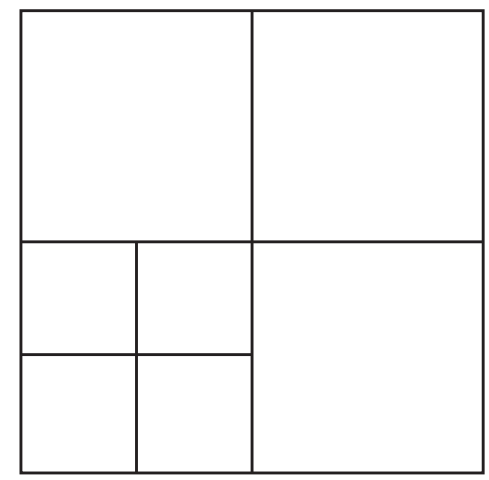

(c)

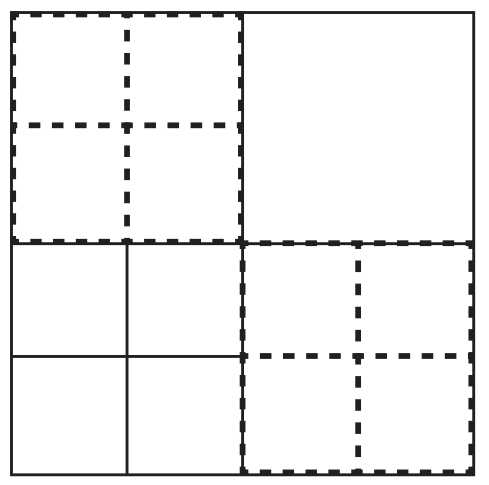

Figure 2. Examples of nested grids (a) and an adaptive grid (b). The level of each grid is denoted by $l$. (c) Virtual cells bounded by dashed lines are added to the adaptive grid (b).

\section{Numerical RESULtS}

\subsection{Flow configuration and initial field}

We consider a two-dimensional periodic channel with a suddenly expanded section, illustrated in Fig. 3. We compute one cell of the periodic channel, for which bifurcation from a steady state to an oscillatory state was observed in [17]. We take the $x_{1}$-axis along the flow direction and the $x_{2}$-axis as the vertical direction. The rectangle shown in dashed lines indicates the boundary of the computational domain $\Omega$. Periodic boundary conditions are used for the computational domain in the $x_{1}$ and $x_{2}$ directions. Note that replacing the successive periodic repetition of the unit cell by a single unit with periodic boundary condition is not equivalent as observed in [17]. The solid line indicates the boundary of the channel wall. The mask function in Eq. (6) is set to $\chi=1$ for the region bounded by the dashed and solid lines, while $\chi=0$ for the fluid region. The shape of the channel is defined by the following three parameters: (i) expansion ratio: $E=D / d$, (ii) aspect ratio of the expanded section: $A=\ell_{e} /(2 D)$, and (iii) nondimensional throat length $s=\ell_{r} / d$. The periodic length $\mathcal{L}$ of the channel is $\mathcal{L}=2(s+E A)$. Here, $E=3, A=7 / 3$ and $s=1 / 2$. The nondimensional length of the solid region in the $x_{2}$-direction is $\ell_{h} / d=3 / 10$. To drive the flow, the forcing term $F_{i}$ is set to be $F_{i}=2(1-\chi) \delta_{i 1} / R e$ and $R e=65$. For details we refer to [18]. The remaining parameters are set as $M a=0.2, \operatorname{Pr}=0.71, \gamma=1.4$ and $T_{\Omega}=1$.

To make an initial field for the three PNS computations in Sec. 4.2 and three CVS computations in Sec. 4.3, we performed a preceding CVS at $\eta=10^{-3}$, until the flow becomes periodic. For the PNS computations, data on the uniform grid are obtained by interpolation of the CVS data.

The number of grid points for the PNS computations is $N_{\text {PNS }}=2^{m} \times 2^{m}$, where $m=8$. The maximum level $L$ of the CVS grid is also $L=m$. Since CVS uses a tree data structure, the maximum number of degrees of freedom is $N_{\text {PNS }} \sum_{l=0}^{m}(1 / 4)^{l}$, which is the summation of the number of grid points at each level $l$.

\subsection{Penalization parameter dependence for penalized Navier-Stokes equations}

To assess the influence of the penalization parameter, we performed three PNS computations for $\eta=$ $10^{-2}, 10^{-3}$ and $10^{-4}$. The corresponding time increments are set to $5 \times 10^{-4}, 5 \times 10^{-4}$ and $5 \times 10^{-5}$, respectively. As discussed theoretically in [19], smaller $\eta$ allows for more accurate solutions for sufficiently fine grids. However, the constraint from the penalization terms becomes more severe than the other constraints as $\eta$ becomes smaller. Thus, it is important to choose a suitable value of $\eta$ for a given grid size, taking into account a balance between the CPU cost and the accuracy of the solutions. We also refer to the discussion in [20]. For the cases of $\eta=10^{-3}$ and $10^{-4}, \Delta t$ is determined by the constraints imposed by $\eta$, while for the case of $\eta=10^{-2}$, $\Delta t$ is determined by the diffusive constraint. 


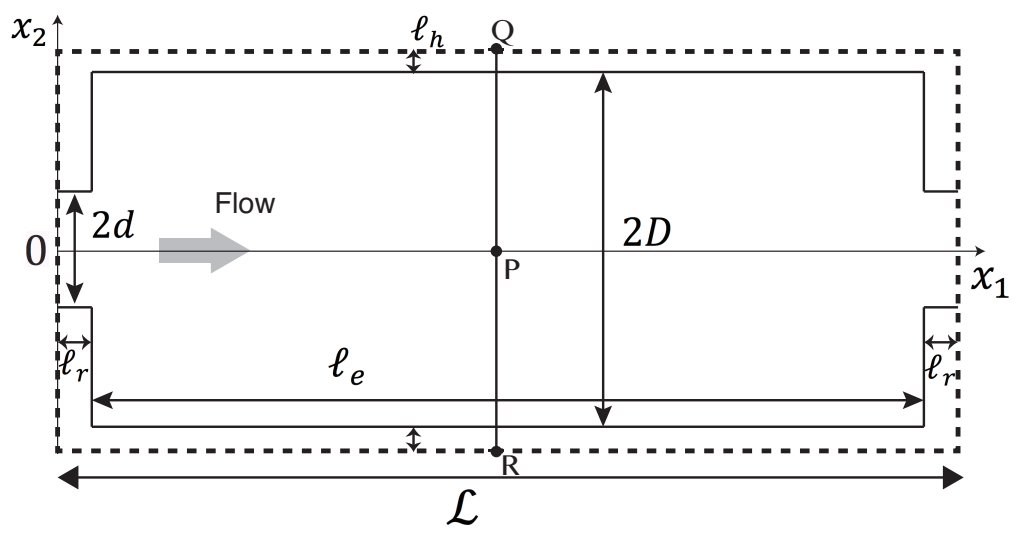

Figure 3. Flow geometry and coordinates.
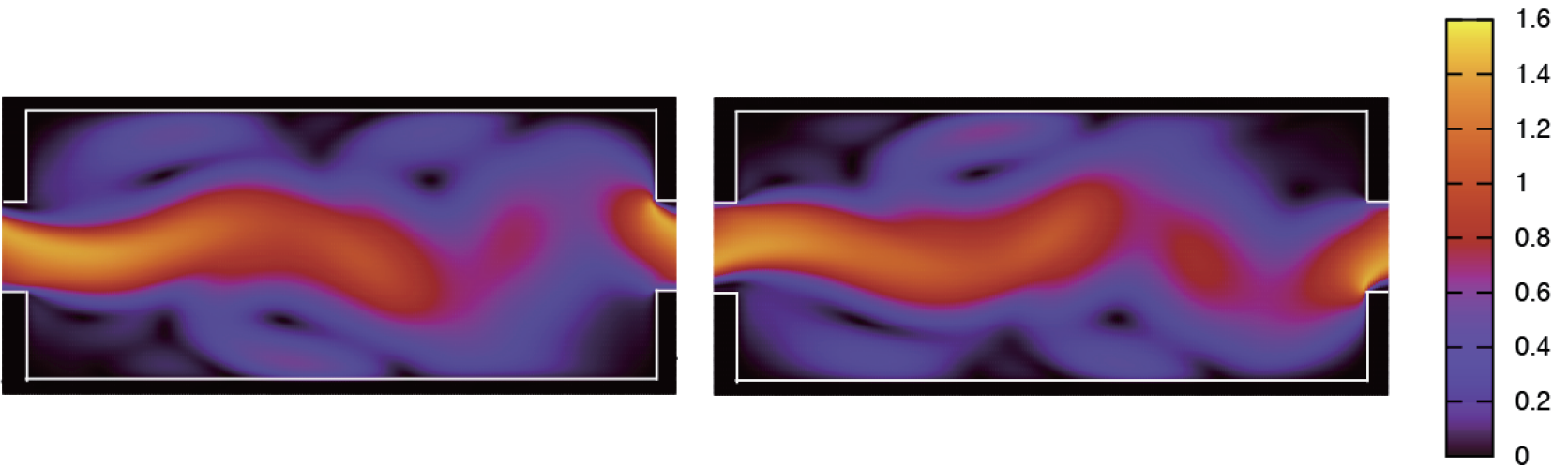

Figure 4. Visualization of $|\boldsymbol{u}|$ for PNS at $t=25$ (left) and $t=35$ (right), where $\boldsymbol{u}=\left(u_{1}, u_{2}\right)$.

The white lines indicate the wall positions.

To get an intuitive idea on the flow field, velocity magnitudes obtained with PNS at $\eta=10^{-3}$ are visualized for two time instants in Fig. 4. We see that wavy structures are formed by the intense velocity regions. The wavy structures seem to oscillate as time increases, as observed for incompressible flow [17].

Figure 5 shows the $\eta$ dependence for the time evolutions of the streamwise velocity $u_{1}$ and the trajectories $\left(u_{1}, u_{2}\right)$ for PNS computations, where $u_{1}$ and $u_{2}$ are measured at the point P indicated in Fig. 3. In Fig. 5 (left), we observe that the curves at $\eta=10^{-3}$ and $\eta=10^{-4}$ overlap well, while the curve at $\eta=10^{-2}$ significantly departs from them after about $t=5$. Figure 5 (right) shows that the curves for $\eta=10^{-3}$ and $\eta=10^{-4}$ agree well with each other. We find that the flows are indeed periodic with a period of about $T_{0} \approx 23.8$. We observe that the curve for $\eta=10^{-2}$ differs from the curves for $\eta=10^{-3}$ and $\eta=10^{-4}$.

Figure 6 shows the $\eta$ dependence of one dimensional cuts of $u_{1}$ along the line $\mathrm{QR}$ given in Fig. 3. It can be seen that two curves for $\eta=10^{-3}$ and $10^{-4}$ overlap well over the whole range of $x_{2}$, while $|\boldsymbol{u}|$ for $\eta=10^{-2}$ is much smaller than $|\boldsymbol{u}|$ for the other two cases around $x_{2} \sim 0$ and -2.3 . Inside the walls of the expanded section, i.e., $\left|x_{2}\right| \geq 3,|\boldsymbol{u}|$ is sufficiently small. This confirms that the volume penalization method models well the walls.

We conclude that $\eta=10^{-3}$ is a suitable value, because (i) the PNS at $\eta=10^{-4}$ is about 10 times more expensive than PNS at $\eta=10^{-3}$ and (ii) the effect of the difference between $\eta=10^{-3}$ and $\eta=10^{-4}$ on the 

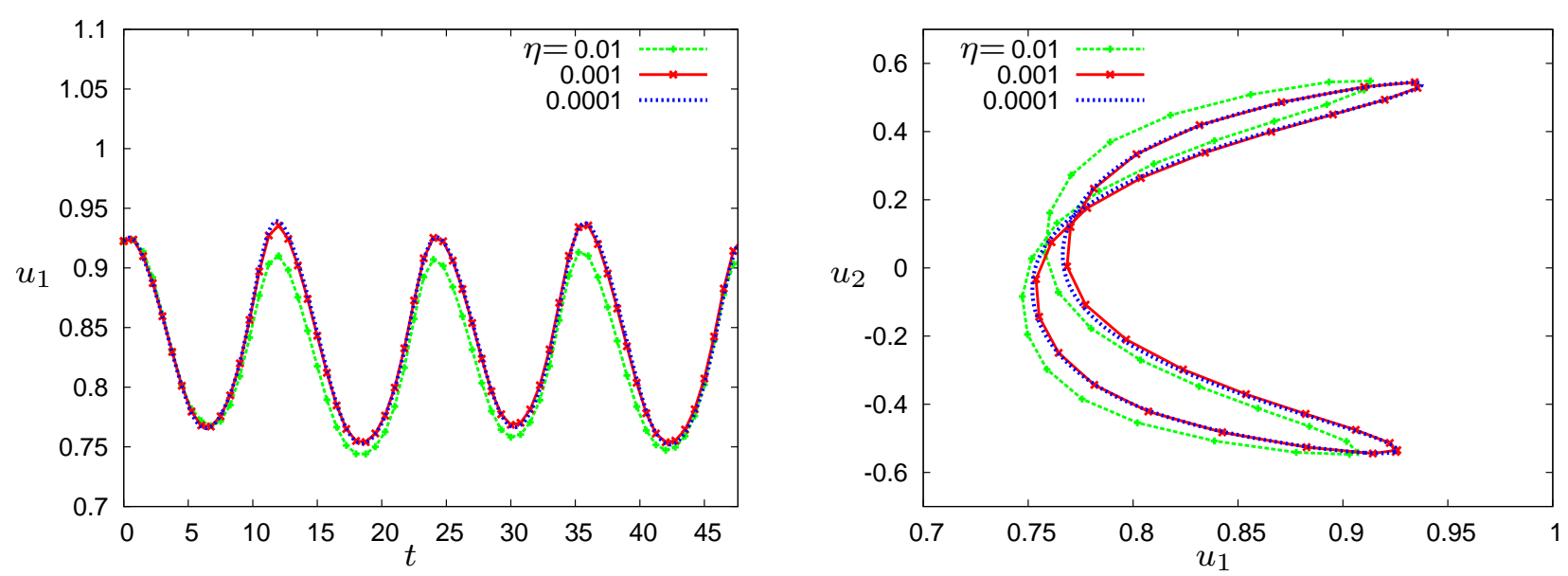

Figure 5. Left: time evolutions of $u_{1}$ at the point $\mathrm{P}$ for $\eta=10^{-2}, 10^{-3}$ and $10^{-4}$. Right: trajectory in the $\left(u_{1}, u_{2}\right)$ plane from $t=24$ to $t=48$.

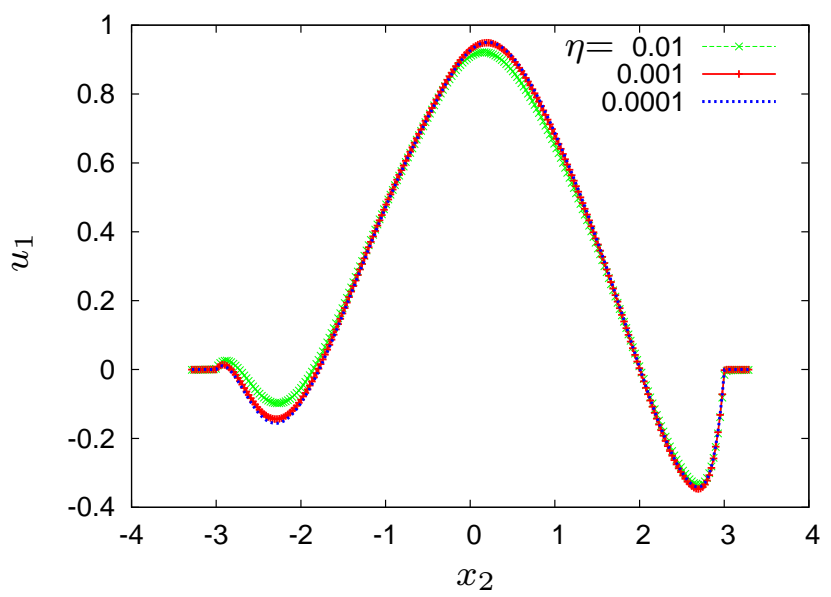

FiguRE 6. One-dimensional cuts of $u_{1}$ at $t=35$ along the line QR.

flow field can be hardly observed. In the following, CVS computations for $\eta=10^{-3}$ are performed for different threshold values. The quality and efficiency of the CVS computations are assessed by comparison with PNS at $\eta=10^{-3}$.

\subsection{Assessment of CVS}

Now we assess the quality and efficiency of CVS, comparing with a reference flow obtained by the PNS computation for $\eta=10^{-3}$. We perform three CVS computations (CVS1, CVS2 and CVS3) using $\eta=10^{-3}$. The threshold value $\epsilon$ used in the wavelet filtering in CVS1, CVS2 and CVS3 is $\epsilon=0.02,0.04$ and 0.08, respectively. 

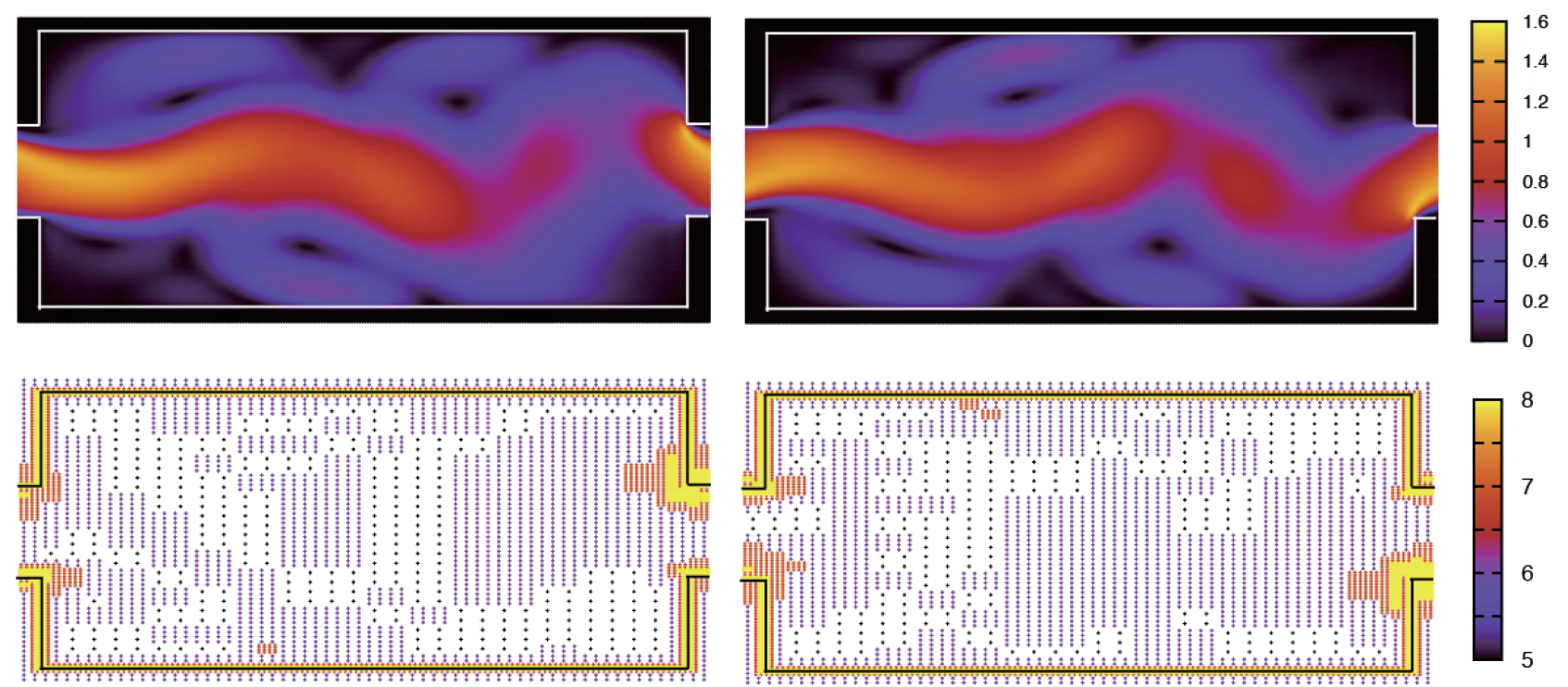

Figure 7. Visualizations of $|\boldsymbol{u}|$ for CVS with $\epsilon=0.04$ at (top, left) $t=25$ and (top, right) $t=35$. Visualizations of the adaptive grids at (bottom, left) $t=25$ and (bottom, right) $t=35$, where the black lines indicate the wall positions. The color bar (bottom, right) indicates grid levels.

We start with visualizing the velocity magnitude at two time instants obtained by CVS2 and the corresponding adaptive grids in Fig. 7. We observe that CVS2 well preserves the wavy structures of the original flow obtained with PNS. There are many grid points at the finest level near the corner of the outlet, which track intense velocity gradients near the corner. Note that the finest level of the grid is $m=8$, which is colored yellow. Away from the corners, the number of grid points is significantly reduced, not only in the fluid region apart from the walls, but also inside the wall regions.

Figure 8 shows the time evolutions of $u_{1}$ and the trajectories in the $\left(u_{1}, u_{2}\right)$ plane at the point $\mathrm{P}$ for the PNS and CVS computations. In Fig. 8 (left), we observe that CVS1 and CVS2 well retain the time evolution of PNS, while CVS3 departs significantly from PNS after about $t=25$. In Fig. 8 (right), it can be seen that the curves for CVS1 and CVS2 are close to the results obtained with PNS. We find that the flows are indeed periodic in time. In contrast, the curve for CVS3 significantly deviates from the curve of PNS. Concerning the one-dimensional cuts of $u$ along the line QR, for the whole $x_{2}$ range, we confirm that CVS1 and CVS2 are in good agreement with PNS and that $|\boldsymbol{u}|$ is sufficiently small inside the walls (figure omitted).

Figure 9 shows the time evolution of the ratio $R=100 N_{\mathrm{CVS}} / N_{\mathrm{PNS}}$, where $N_{\mathrm{CVS}}$ consists of two parts; one is the number of degrees of freedom (DoF) of the adaptive mesh on which the flux computations are carried out, and the other is the number of DoF inside the tree, which are necessary to perform the multiresolution analysis. The latter causes overhead of CVS, that does not exist in the PNS computation. However, if the overhead is not so significant, CPU gain can be larger than memory gain. Figure 9 shows, as expected, that most coefficients are retained by CVS1 (about 31\%), followed by CVS2 (about 26\%), and then CVS3 (22\%). Table 1 summarizes the computational cost of each computation and the relative error obtained from Fig. 8 (left). The relative error is a measure of the predictability of CVS, which is defined in the caption. We conclude that CVS1 and CVS2 are reasonable choices taking into account the balance between CPU time and predictability of the flow. In CVS, the deterministic predictability of CVS becomes better for smaller $\epsilon$, as expected. However, the computational cost of CVS with small $\epsilon$ is large. Therefore, the balance between computational cost and predictability is important. 

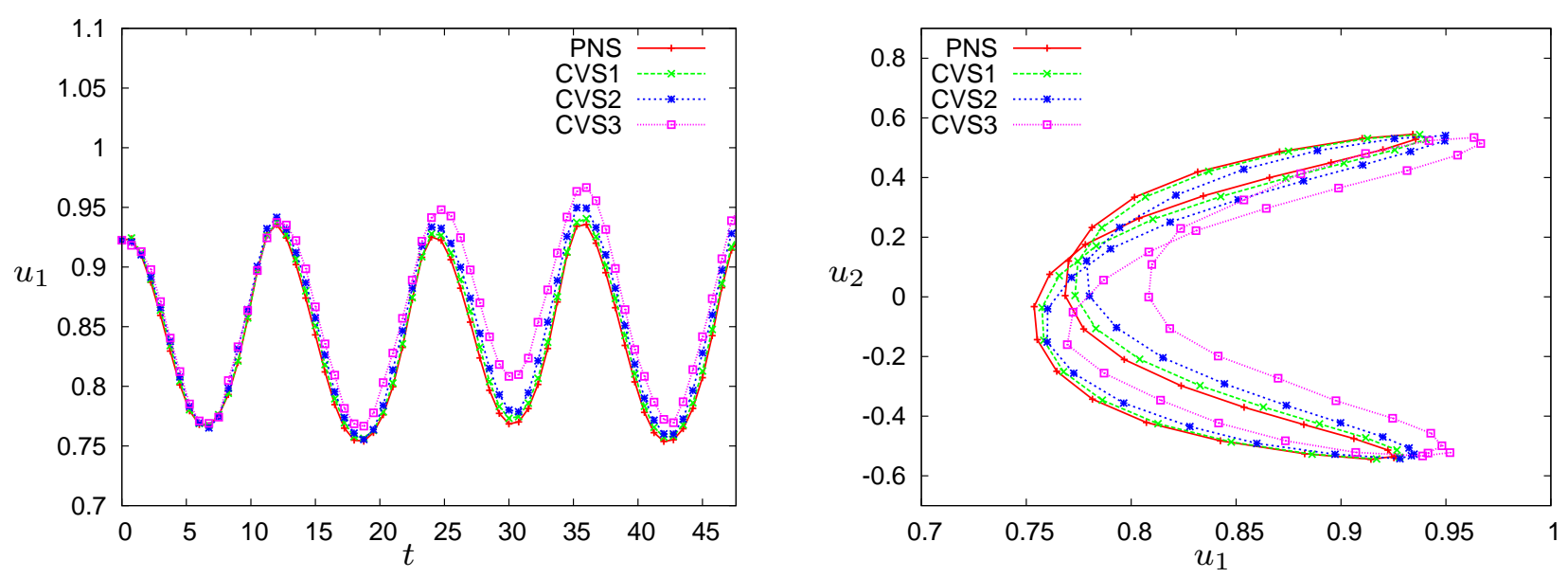

Figure 8. Time evolutions of $u_{1}$ (left) and trajectories in the $\left(u_{1}, u_{2}\right)$ plane at the point $\mathrm{P}$ (right).

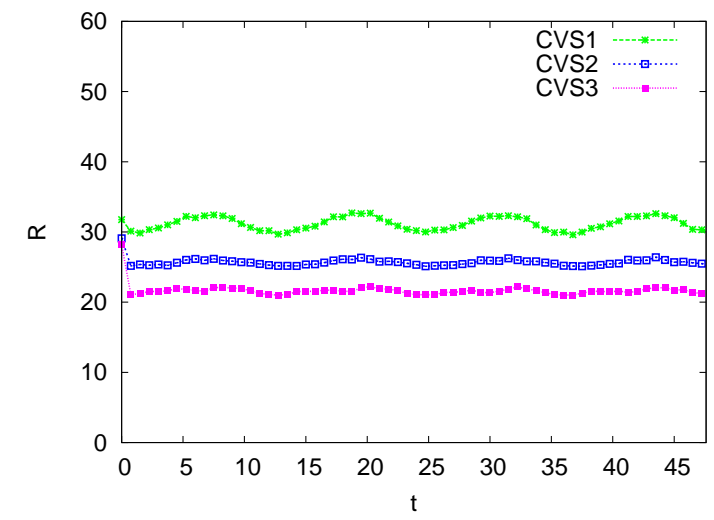

Figure 9 . Time evolutions of $R=100 N_{\mathrm{CVS}} / N_{\mathrm{PNS}}$ for different thresholds.

\section{Conclusion and Perspectives}

We have developed an adaptive multiresolution simulation method, called CVS, to compute weakly compressible flow bounded by walls with complex geometry, using biorthogonal wavelets and a finite volume approach. A volume penalization method has been employed to compute the flow in a Cartesian geometry and to impose the boundary conditions. The quality and efficiency of the method have been assessed for the flow in a periodic channel with a suddenly expanded cross section, comparing with a reference flow obtained by the non-adaptive FV simulation (PNS).

We found that the CPU time of CVS is about five times smaller than that of PNS, while preserving the deterministic predictability with 25 to $30 \%$ of the number of degrees of freedom compared to that of the PNS 


$\begin{array}{lcccc}\text { Method } & \text { CPU time } & \text { CPU (CVS/PNS) } & \text { Memory } & \text { Relative error } \\ \text { PNS } & 22 \text { h } 21 \mathrm{~min} & - & - & - \\ \text { CVS1 } & 5 \text { h } 3 \text { min } & 22.6 \% & 31.1 \% & 1.1 \% \\ \text { CVS2 } & 4 \text { h } 2 \text { min } & 18.0 \% & 25.6 \% & 2.7 \% \\ \text { CVS3 } & 3 \text { h } 13 \mathrm{~min} & 14.4 \% & 21.5 \% & 6.5 \%\end{array}$

TABLE 1. Computational costs for the PNS and CVS computations. Memory compression is defined by time average of $R$. Relative error is defined by the maximum value of $100 \mid u_{1}^{\mathrm{CVS}}-$ $u_{1}^{\mathrm{PNS}}|/| u_{1}^{\mathrm{PNS}} \mid$ at the point $\mathrm{P}$ for $0<t<47.6$.

computation. The number of the grid points in CVS is significantly reduced in the fluid region apart from the wall and inside the wall region.

The efficiency of the present CVS can be improved further using spatially variable time stepping method [21]. This CVS would allow for adaptivity both in space and time, which can lead to further speed-up of CPU time. The application of CVS to reacting flow may be promising, because reacting flow is strongly multiscale both in space and time $[22,23]$. The assessment of adaptive CVS for turbulent flows bounded by walls of complex geometries is also an important issue. In [24], it was shown that wavelet representations are more efficient than non-adaptive representations for isotropic turbulent flow, as the Reynolds number increases. It is thus expected that adaptive CVS is an attractive tool for high Reynolds number flows bounded by walls in complex geometries.

\section{ACKNOWLEDGMENTS}

The computations were carried out on the CX system at the Information Technology Center of Nagoya University. This work was partially supported by JSPS KAKENHI Grant Numbers (B)24760063, (S)24224003 and (A)25247014. K.S. thankfully acknowledges financial support from ANR, contract SiCoMHD. M. O. D. thankfully acknowledges financial support from Ecole Centrale de Marseille (ECM) for a visiting position, Fundação de Amparo a Pesquisa do Estado de São Paulo (FAPESP), and CNPq - the Brazilian Research Council, Brazil. K.S., K.Y. and N.O. acknowledge the CIRM, Luminy, for hospitality during the 2014 CEMRACS summer program on "Numerical modeling of plasmas".

\section{REFERENCES}

[1] G. De Stefano and O. V. Vasilyev, Wavelet-based adaptive simulations of three-dimensional flow past a square cylinder, $J$. Fluid Mech., 748:433-456, 2014.

[2] E. B. Dymkoski, N. Kasimov and O. V. Vasilyev, A characteristic based volume penalization method for general evolution problems applied to compressible viscous flows, J. Comput. Phys., 262:344-357, 2014.

[3] O. Boiron, G. Chiavassa and R. Donat, A high-resolution penalization method for large Mach number flows in the presence of obstacles, Computers \& Fluids, 38(3):703-714, 2009.

[4] K. Schneider, Immersed boundary methods for numerical simulation of confined fluid and plasma turbulence in complex geometries: a review, J. Plasma Phys., doi:10.1017/S0022377815000598, in press, arXiv:1508.04593, 2015.

[5] R. Deiterding, M. Domingues, S. Gomes and K. Schneider, Comparison of adaptive multiresolution and adaptive mesh refinement applied to simulations of the compressible Euler equations, Preprint, 06/2015, submitted.

[6] R. Deiterding, M. O. Domingues, S. M. Gomes, O. Roussel and K. Schneider, Adaptive multiresolution or adaptive mesh refinement? A case study for 2D Euler equations, ESAIM: Proceedings, 29:28-42, 2009.

[7] K. Schneider and O. Vasilyev, Wavelet Methods in Computational Fluid Dynamics, Annu. Rev. Fluid Mech., 42:473-503, 2010.

[8] M. Domingues, S. Gomes, O. Roussel and K. Schneider, Adaptive multiresolution methods, ESAIM: Proceedings, 34:1-96, 2011.

[9] Y. Asako, T. Pi, S. E. Turner and M. Faghri, Effect of compressibility on gaseous flows in micro-channels, Int. J. Heat Mass Transfer, 46:3041-3050, 2003.

[10] C. S. Peskin, The immersed boundary method, Acta Numer., 11(1):479-517, 2002. 
[11] O. Roussel and K. Schneider, Coherent Vortex Simulation of weakly compressible turbulent mixing layers using adaptive multiresolution methods, J. Comput. Phys., 229(6):2267-2286, 2010.

[12] S. Mallat, A wavelet tour of signal processing, Third Edition: The Sparse Way, Academic Press, 2010.

[13] M. Farge, G. Pellegrino and K. Schneider, Coherent vortex extraction in 3d turbulent flows using orthogonal wavelets, Phys. Rev. Lett., 87:45011-45014, 2001.

[14] M. Farge, K. Schneider and N. Kevlahan, Non-Gaussianity and coherent vortex simulation for two-dimensional turbulence using an adaptive orthonormal wavelet basis, Phys. Fluids, 11:2187-2201, 1999.

[15] M. Farge and K. Schneider, Coherent Vortex Simulation (CVS), a semi-deterministic turbulence model using wavelets, Flow, Turbul. Combust., 66:393-426, 2001.

[16] D. Kolomenskiy and K. Schneider, A Fourier spectral method for the NavierStokes equations with volume penalization for moving solid obstacles, J. Comput. Phys., 228(16):5687-5709, 2009.

[17] M. Takaoka, T. Sano, H. Yamamoto, and J. Mizushima, Convective instability of flow in a symmetric channel with spatially periodic structures, Phys. Fluids, 21(2):024105, 2009.

[18] Y. Morinishi, Skew-symmetric form of convective terms and fully conservative finite difference schemes for variable density low-Mach number flows, J. Comput. Phys., 229(2):276-300, 2010.

[19] P. Angot, C. H. Bruneau and P. Fabrie, A penalization method to take into account obstacles in incompressible viscous flows, Numer. Math., 81:497-520, 1999.

[20] R. Nguyen van yen, D. Kolomenskiy and K. Schneider, Approximation of the Laplace and Stokes operators with Dirichlet boundary conditions through volume penalization: a spectral viewpoint, Numer. Math., 128:301-338, 2014.

[21] M. O. Domingues, S. M. Gomes, O. Roussel and K. Schneider, Space-time adaptive multiresolution methods for hyperbolic conservation laws: Applications to compressible Euler equations, Appl. Numer. Math., 59(9):2303-2321, 2009.

[22] M. Duarte, Z. Bonaventura, M. Massot, A. Bourdon, S. Descombes and T. Dumont, A new numerical strategy with space-time adaptivity and error control for multi-scale streamer discharge simulations, J. Comput. Phys., 231(3):10021019, 2012.

[23] O. Roussel and K. Schneider, Adaptive multiresolution computations applied to detonations, Z. Phys. Chem., 229(6):931-953, 2015.

[24] N. Okamoto, K. Yoshimatsu, K. Schneider, M. Farge and Y. Kaneda, Coherent vortices in high resolution direct numerical simulation of homogeneous isotropic turbulence : A wavelet viewpoint, Phys. Fluids, 19:115109, 2007. 